\title{
Photonic Network Design Based on Reference Circuits
}

Ernst-Jürgen Bachus, Michael Eiselt, Kai Habel, Klaus-Dieter Langer Heinrich-Hertz-Institut für Nachrichtentechnik Berlin GmbH, Berlin, Germany Ernst-Ulrich Scheuing, Friedrich-Christian Tischer

Bosch Telecom GmbH, Backnang, Germany

\begin{abstract}
The ever increasing demand for network capacity is driving new technologies into realization. One of these are future transparent Photonic Networks, providing considerably more functions than plain transmission only through independent routing of signals by means of Wavelength Division Multiplexing. However, the analogue nature of such Photonic Networks results in an accumulating degradation of the transported optical signals and prevents the application of simple design rules.
\end{abstract}

The objectives of this presentation are to clarify specific terms like transparency and transverse compatibility, and then to derive guidelines as a first approach to an engineered Photonic Network. These guidelines are applied to the planning of a core network with 8 and 16 wavelength channels per link and verified by first numerical results.

Complementary to a layered network architecture, our methodology is based on the use of a specific reference configuration. Degradation effects like amplifier noise, chromatic and polarization-mode dispersion, non-linear self phase modulation are covered as well as node crosstalk and the impact of optical frequency misalignments. Based on ITU-T recommendations, a classification of ranges of bitrates and other preliminary specifications, our method allows to assemble a general Photonic Network from its elements in a bottom-up scheme.

As a result, we show that Photonic Networks could exhibit transparent optical paths, ranging from 400 to several thousands of kilometres. A number of 16 wavelength channels at individual bitrates of up to $10 \mathrm{Gbit} / \mathrm{s}$ traversing a couple of crossconnecting nodes can be implemented, taking into account present-day optical components like amplifiers, standard fibres, multiplexers and demultiplexers, fibre switches as well as dispersion compensating techniques. The potential benefits of such networks are to be seen in their inherent high capacity and in a high degree of flexibility, supporting various applications.

Considering the results obtained so far, it can be concluded that a country of the size of Germany could be covered by a transparent Photonic Network.

\section{Introduction}

Fibre networks with considerably more functions than plain transmission are called Photonic Networks. Wavelength Division Multiplexing (WDM) is one of the features of such networks for multiplexing and optical routing of independent signals. This makes it possible to form an "underlying" network which supports other networks. The achievable transparency, high capacity, good transmission quality and robustness make Photonic Networks attractive. Insofar, many field trials are under way around the world.

However, a fundamental problem of Photonic Networks results from its analogue nature.

The transported optical signals suffer from continuous and accumulating degradation on their way through the fibre network. This degradation is not yet well known in all details and prevents the application of simple design rules for such networks.

This contribution is a first step to develop guidelines for realising Photonic Networks. Accompanying quantitative calculations indicate that this approach seems to be principally reasonable. 


\section{Photonic Networks and the Role of WDM}

A Photonic Network is here understood as a network

- which consists of interconnecting lines and network nodes

- which is suitable for optical transmission with high capacity

- which is based on widespread use of WDM

- which allows initial configuration, later reconfiguration due to changing traffic demands, and restoration in case of line failures

- which provides maximal transparency for present-day and future services and signal types, including "leased lines" with parameters still to be defined

- which processes the signals in the optical domain

- which is based on the joint use of space and frequency switching

- which has integrated supervision and management facilities with technical standards comparable to those of SDH equipment

- which provides "embedded" transport of OAM information within the network

- which is compatible with existing transmission equipment regarding

- optical interfaces

- switching times and

- network management.

In short, a Photonic Network is a sophisticated and switchable replacement of an optical fibre plant.

\subsection{Analogue Character and Reference Configuration}

In digital networks no substantial signal degradation occurs during transmission and within the equipment. It is therefore sufficient to describe it by means of the "layered network architecture" and by "functions" in every layer.

On the other hand, and seen from many aspects like

- power levels

- dispersion

- frequency distortion

- noise accumulation and

- crosstalk

the Photonic Network has clearly features of an analogue network. Quasi-analogue transmission leads to an accumulating degradation, even if all components work as assumed.

The layered network approach is therefore less suitable and not sufficient to describe the analogue effects. 


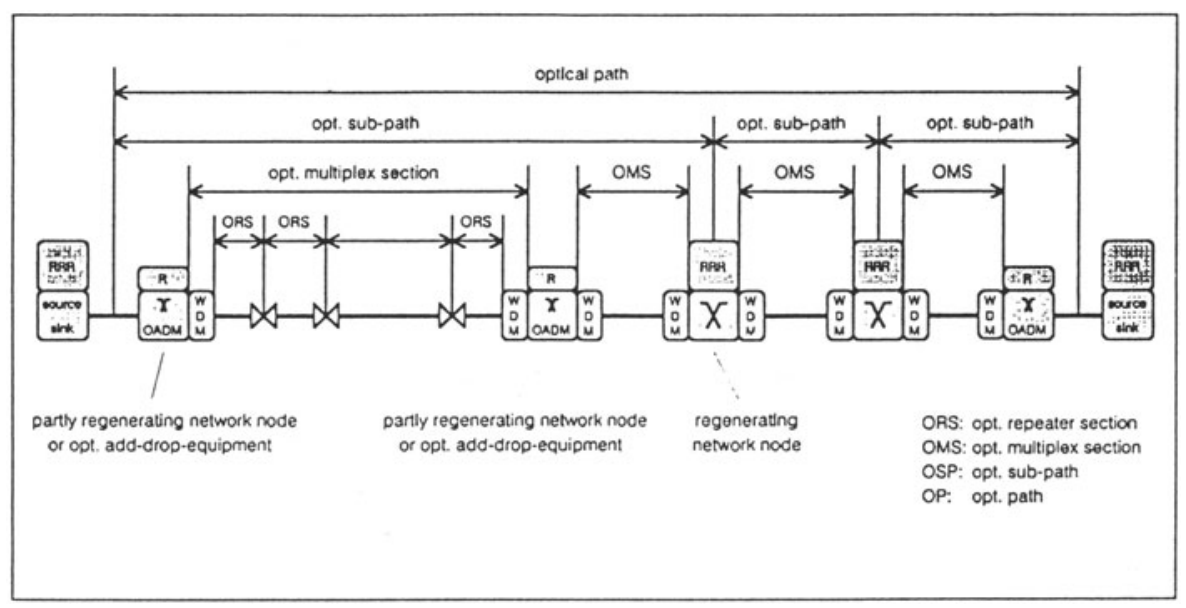

Fig. 1: Photonic Network Reference Circuit

In addition, a reference configuration is proposed, with

- partly regenerating optical crossconnects

- optical add/drop multiplexers

- fully regenerating crossconnects

- nonregenerating repeaters or amplifiers, and with

- optical source and sink, which are normally outside the network.

This reference configuration defines

- an "optical wavelength path" which goes from the source to the sink reference points

- "optical subpaths", terminated by fully regenerating crossconnects

- "optical multiplex sections", ranging from crossconnect to crossconnect or optical add-drop-multiplexer, and

- "optical repeater sections" between nonregenerative repeaters.

The transparent optical sub-path consists of cascaded optical repeater sections and multiplex sections. Cascading of several sub-paths is not critical due to the complete regeneration at the end of each sub-path.

The basic parameters for a first calculation of an optical repeater section are summarised in Table 1. 


\begin{tabular}{|c|c|c|c|}
\hline bitrate class & class 1 & class 2 & class 3 \\
\hline maximum bitrate & 0.7 & 2.5 & 10 \\
\hline \multirow[t]{3}{*}{ application } & up to STM-4 & up to STM-16 & up to STM-64 \\
\hline & up to $O C-12$ & up to $O C-48$ & up to OC-192 \\
\hline & $34 / 140 / 565 \mathrm{Mbit} / \mathrm{s}$ & & \\
\hline standard fibre acc. ITU-T G.652 & yes & yes & $\begin{array}{c}\text { yes, plus dispersion } \\
\text { compensation }\end{array}$ \\
\hline target distances (ORS length) & $120 \mathrm{~km}$ & $120 \mathrm{~km}$ & $80 \mathrm{~km}$ \\
\hline ORS attenuation $(0.275 \mathrm{~dB} / \mathrm{km})$ & $33 \mathrm{~dB}$ & $33 \mathrm{~dB}$ & $22 \mathrm{~dB}$ \\
\hline EDFA noise figure (assumed) & $5 \mathrm{~dB}$ & $5 \mathrm{~dB}$ & $5 \mathrm{~dB}$ \\
\hline maximum launched power, total & $+17 \mathrm{dBm}$ & $+17 \mathrm{dBm}$ & $+17 \mathrm{dBm}$ \\
\hline per channel ( 8 channels) & $+8 \mathrm{dBm}$ & $+8 \mathrm{dBm}$ & $+8 \mathrm{dBm}$ \\
\hline per channel ( 16 channels) & $+5 \mathrm{dBm}$ & $+5 \mathrm{dBm}$ & $+5 \mathrm{dBm}$ \\
\hline
\end{tabular}

\section{Table 1: Basic Reference Circuit Parameters}

\subsection{Network Evolution}

The final goal to implement a Photonic Network may be reached by evolutionary introduction of WDM to existing fibre networks in several steps:

- in a first step, for capacity enlargement in point-to-point links

- in a second step, for flexibility and protection in WDM rings

- in a final step, as a general means to enlarge capacity and flexibility of the network.

The first form of application leads to rather simple WDM equipment, the second and the third lead to much more stringent requirements, as regards frequency stability, optical frequency and dispersion equalization, power level management, and crosstalk.

\subsection{Transparency}

The original idea of transparency was to have a network into which one could launch any optical signal and nothing in the network would interfere with its transmission, and only the terminal equipment would determine the limitations of the signal.

At a closer look we can see that there are limits to almost every parameter of the signal. Many of those limits depend on its physical properties and so full transparency cannot be achieved.

A Photonic Network should provide transparency for all types of signals up to a feasible upper limit according to the state of the art, i.e. up to the order of $10 \mathrm{Gbit} / \mathrm{s}$.

The demand for transparency is to be limited regarding several aspects:

- the characteristics of the signals to be transmitted

- fibre nonlinearities and power levels

- crosstalk in wavelength selective components and switches

- the maximum length of regenerator and multiplex sections

- the accumulated noise and hence the signal-to-noise-ratio (which depends on the bit rate)

- the physical properties of the fibre like frequency-dependent attenuation and dispersion compensation

- the need to specify standard wavelengths of the system in order to guarantee interworking. 


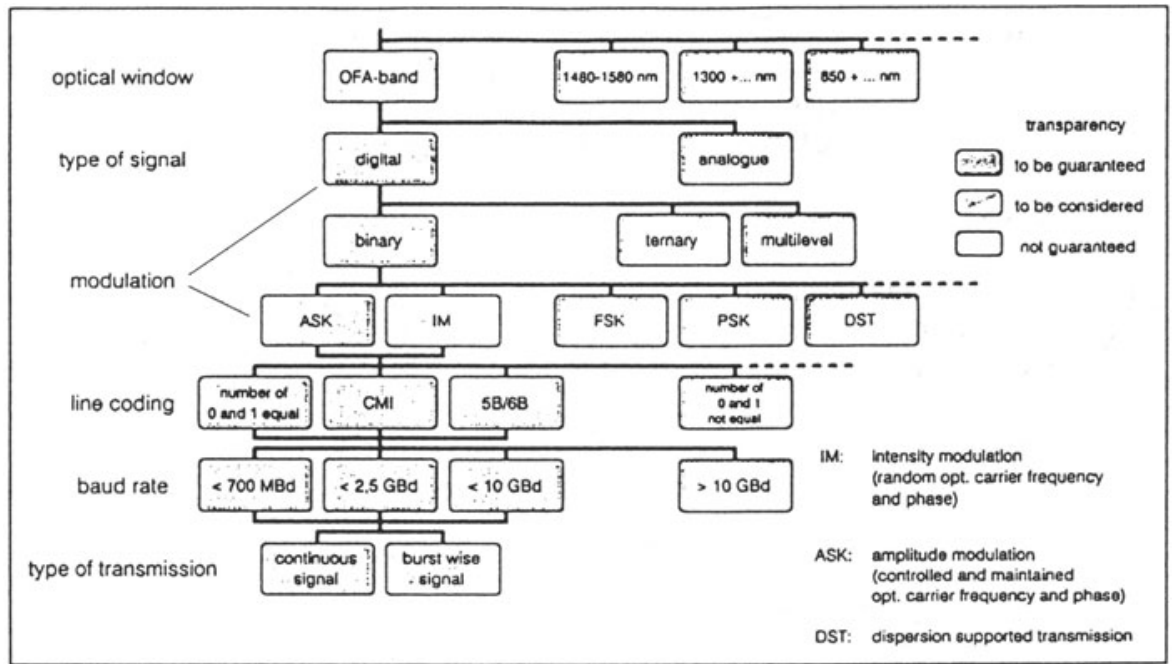

Fig. 2: Transmission Procedures and Transparency

Our definition of transparency is largely in line with an already known proposal:

- optical window is the OFA band

- the type of signal to be transported is digital

- binary modulation is assumed

- the modulation formats are ASK, and IM,

- statistically scrambled signals and CMI or $5 \mathrm{~B} / 6 \mathrm{~B}$ coded signal are transmitted

- line baud rate is up to $10 \mathrm{Gbit} / \mathrm{s}$

- continuous signals are transported, burstwise signals are excluded on account of potential difficulties

- optical frequencies and their stability are to be considered.

The shaded issues are essential and must be met. Other types of signals may also be transported, but this is not essential. Note that analogue signals like TV are explicitly excluded.

The finally agreed degree of transparency should be clarified very early, because it influences many parameters.

Due to economical reasons, it seems not the best way to make every link suitable for signals up to the highest bitrate. Achievable link lengths, number of cascadable amplifiers, receiver sensitivity, and so on, depend on the maximum bitrate. It is therefore advisable to distinguish between several "classes" of links.

\subsection{Bit Rate Classification}

We take the channel bandwidth as the basis for a bit rate classification:

- class 1, which is suitable for signals up to $700 \mathrm{Mbit} / \mathrm{s}$ per channel

-class 2, suitable for signals up to $2.5 \mathrm{Gbit} / \mathrm{s}$ per channel

-class 3 , for signals up to $10 \mathrm{Gbit} / \mathrm{s}$ per channel.

Higher classes should also be usable for transmission of signals of lower classes. It is however to be studied if "downgrading" imposes higher requirements on the equipment. 


\subsection{Potential Frequency Allocation}

Frequency allocations for WDM systems should

- provide as much commonality as feasible

- take state-of-the-art amplifier, transmitter and filter parameters into account

- be based on existing systems and frequency allocations

and

- leave a certain freedom for individual realizations and technical progress.

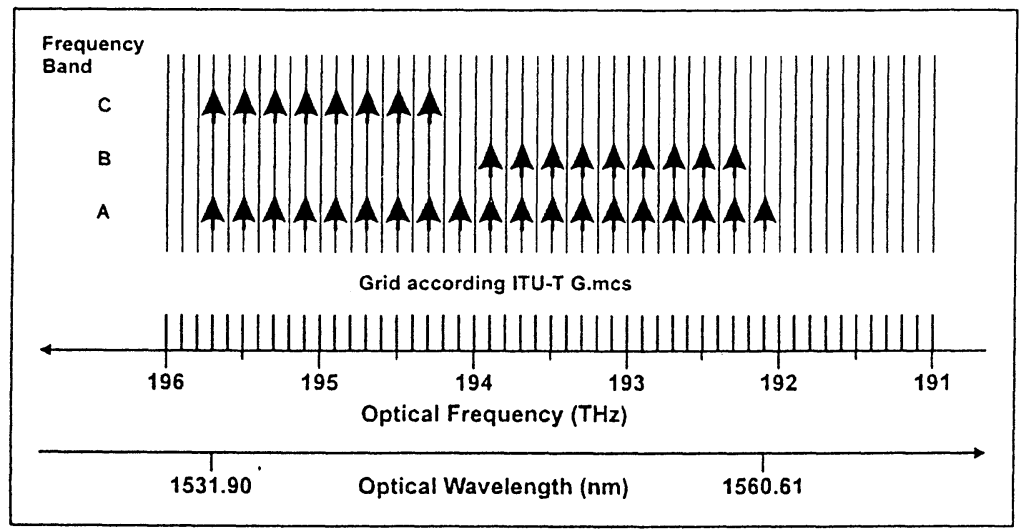

Fig. 3: Wavelength / Frequency Channel Allocation

This proposal is based on a $100 \mathrm{GHz}$ grid within the $1550 \mathrm{~nm}$ band, with the grid "anchored" at 193.1 THz. In view of optical amplifiers and filters, use of band A with $200 \mathrm{GHz}$ spacing of channels is foreseen. Preferred subbands are B (with 9 channels), and C (with 8 channels). Even spacing of channels is assumed for use with standard single-mode fibres. Uneven spacing must be mainly used with fibres according to G.653.

\subsection{Dominant Penalty Principle}

For our calculations we use the "dominant penalty" principle. All degradation effects for cascaded link elements and nodes are treated separately. The " $1 \mathrm{~dB}$ power penalty" criterion is used for each single effect, and the maximum number of cascaded elements is calculated. The dominant degradation effect is determined by the smallest number of cascadable elements. This is taken as the upper limit for the whole system. By lowering the dominant effect, the whole system could be improved.

In the next steps, these principles are applied to the links and to the nodes. The result is an estimation of the network size and capacity which could be built on the basis of the possible number of cascaded units.

The last step is an evaluation of the achieved path performance, considering all remaining effects which are not included in the preceding procedure.

\subsection{Link Dimensioning}

The maximum length of a transparent sub-path is limited by different degradation effects. Strong degradation effects lead to short sub-path lengths.

A common approach is to obtain the longest possible transparent sub-path length by optimising all link parameters. Our approach is cascading identical sections with given target distances, which are in line with ITU-T practice, as shown in the reference configuration. The drawback is of course that we do not necessarily obtain the longest possible sub-path lengths. However, benefits are: 
- a simplified link design by cascading identical repeater sections

- separation of degradation effects by treating all degradations separately with the "1-dB-penalty" criterion and then applying the simple "dominant penalty" principle

- obtaining a single parameter, the number of maximum cascadable sections

- getting a certain degree of modularity.

\subsection{Link Degradation Effects}

A sub-path suffers mainly from the following degradation effects of accumulating character:

- amplifier noise

- polarization mode dispersion

- chromatic dispersion

- non-linear self phase modulation (SPM) in combination with chromatic dispersion

The amplifier noise contribution e.g. can be calculated by means of the formulas given in a recent draft of an ITU-recommendation.

Measurements on installed fibres give actual PMD values. PMD sets hard limits for the maximum transmissible bitrate; this justifies again the bitrate classification.

The non-linear SPM is a fibre effect which depends on the actual optical power and bitrate, resulting in a broadening of the optical spectrum. A broader spectrum suffers more from chromatic dispersion, therefore the combined effect of SPM and chromatic dispersion has to be considered.

Other non-linear effects like

- stimulated Brillouin scattering

- stimulated Raman scattering

- cross-phase modulation

- four-wave mixing

have been calculated to be negligible on standard fibres with power levels below $+15 \mathrm{dBm}$.

Basic Transmission System Parameters:

\begin{tabular}{|c|c|c|c|c|c|c|}
\hline Bitrate class & \multicolumn{2}{|c|}{ class 1} & \multicolumn{2}{|c|}{ class 2} & \multicolumn{2}{|c|}{ class 3} \\
\hline Maximum bitrate & \multicolumn{2}{|c|}{$0.7 \mathrm{Gbit} / \mathrm{s}$} & \multicolumn{2}{|c|}{$2.5 \mathrm{Gbit} / \mathrm{s}$} & \multicolumn{2}{|c|}{$10 \mathrm{Gbit} / \mathrm{s}$} \\
\hline Target ORS-distance & \multicolumn{2}{|c|}{$120 \mathrm{~km}$} & \multicolumn{2}{|c|}{$120 \mathrm{~km}$} & \multicolumn{2}{|c|}{$80 \mathrm{~km}$} \\
\hline Number of wavelength channels & 8 & 16 & 8 & 16 & 8 & 16 \\
\hline ORS launched power per channel & $8 \mathrm{~dB}$ & & $8 \mathrm{dE}$ & $5 \mathrm{dE}$ & $d B$ & \\
\hline
\end{tabular}

Maximum Number of Repeater-Sections (ORS) due to Individual Limiting Effects:

\begin{tabular}{|l|c|c|c|c|c|c|}
\hline Amplifier noise accumulation & 44 & 22 & 12 & 6 & 39 & 19 \\
\hline Combined self-phase modulation and dispersion & 3476 & 6934 & 221 & 439 & 16 & 32 \\
\hline Polarization mode dispersion (0.1 ps/sqrt(km)) & 21300 & 21300 & 1333 & 1333 & 125 & 125 \\
\hline Polarization mode dispersion (0.5 ps/sqrt(km)) & 853 & 853 & 53 & 53 & 5 & 5 \\
\hline Four Wave Mixing & 125 & 400 & 125 & 400 & 125 & 400 \\
\hline
\end{tabular}

Table 2: Maximum Number of Optical Repeater Sections

Table 2 shows the number of achievable repeater sections as a result of the first calculations.

The shaded numbers indicate the dominant degradation effect. From these, the maximum length of sub-paths is easily obtained. 


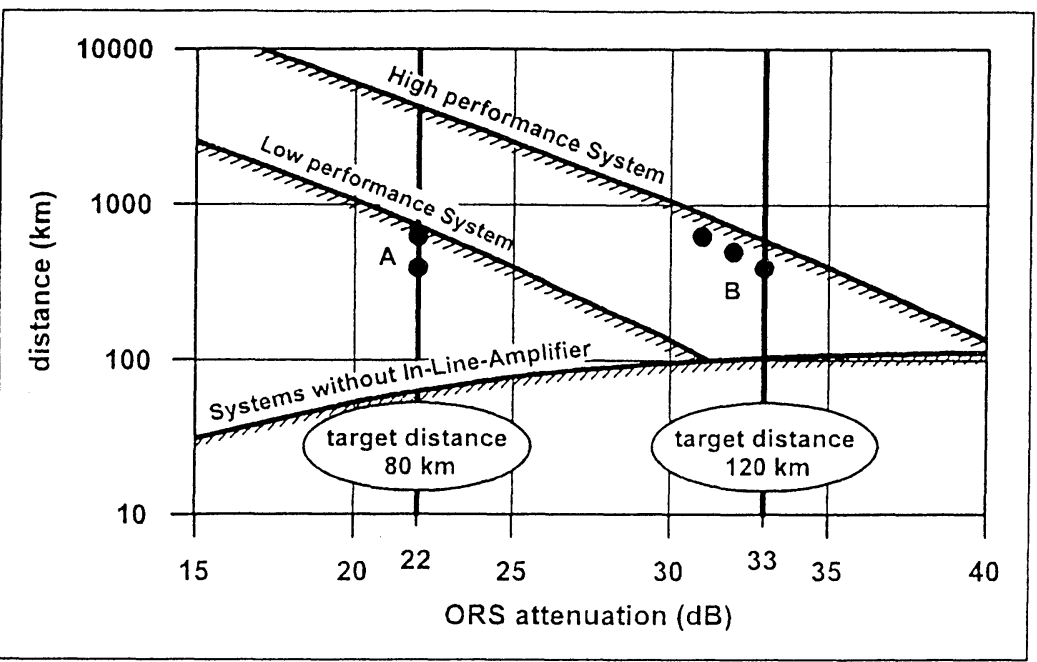

Fig. 4: Achievable Sub-Path Length as a Function of the Repeater Section Attenuation

The boundaries mark systems without in-line amplifiers, and systems with in-line amplifiers of low and high performance.

The following can be concluded:

- the range of sub-path length varies up to one decade between systems with amplifiers of low and high performance

- reducing the ORS span length or attenuation (i.e. the target distance) improves the achievable total distance.

It is however to be considered to stick to ITU-values. 


\section{Network Node}

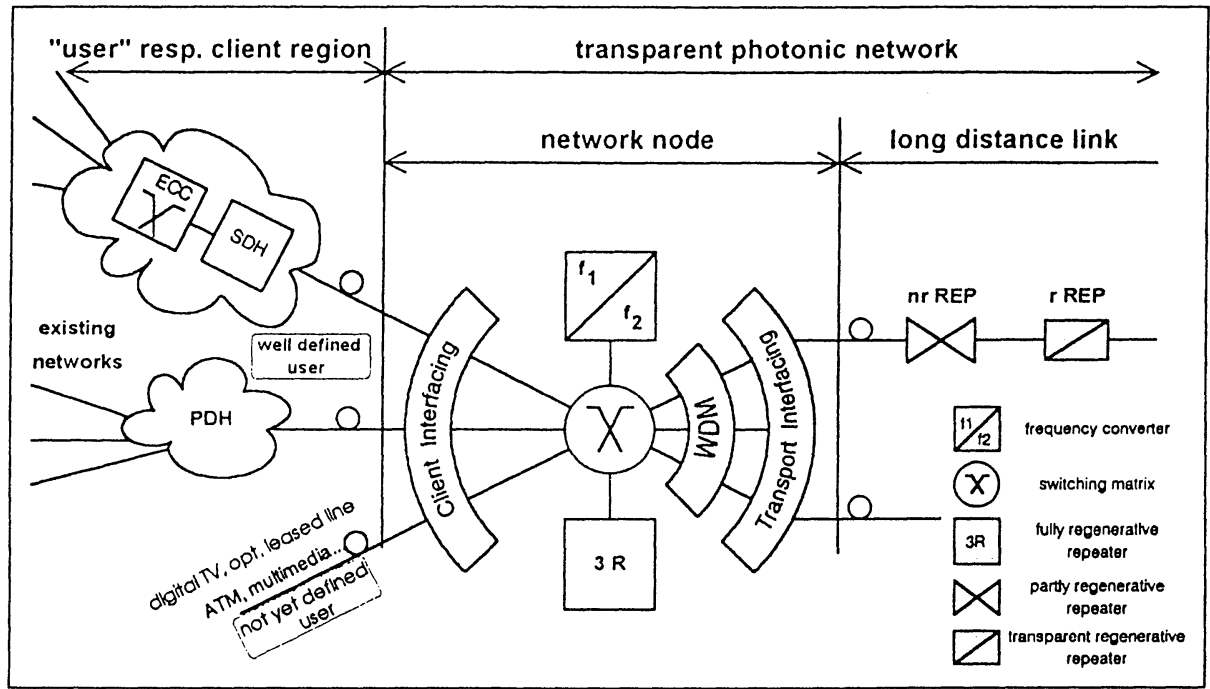

Fig. 5: Network Node

A network node consists principally of a switching matrix which is "shielded" against the environment by interfacing circuits, working together with $3 R$-regenerators and frequency converters as required. $A$ distinction could be made between

- transport nodes with transport interfaces, which are located within the switchable network, and

- access nodes, which provide client interfaces for access to the network.

In practice, every real node will be a mixture of both, because normally network nodes will be at locations where access is also necessary.

\subsection{Node Performance}

The maximum number of cascadable repeater sections in an optical sub-path has been evaluated. Now we replace optical amplifiers by transparent non-regenerating nodes. Each node is considered to be equivalent to one amplifier in view of degradation effects like noise accumulation. This substitution then causes no changes in the sub-path noise performance. The insertion loss can be easily compensated by additional amplifiers, as long as their noise contribution is negligible.

Crosstalk, however, can cause serious problems. 


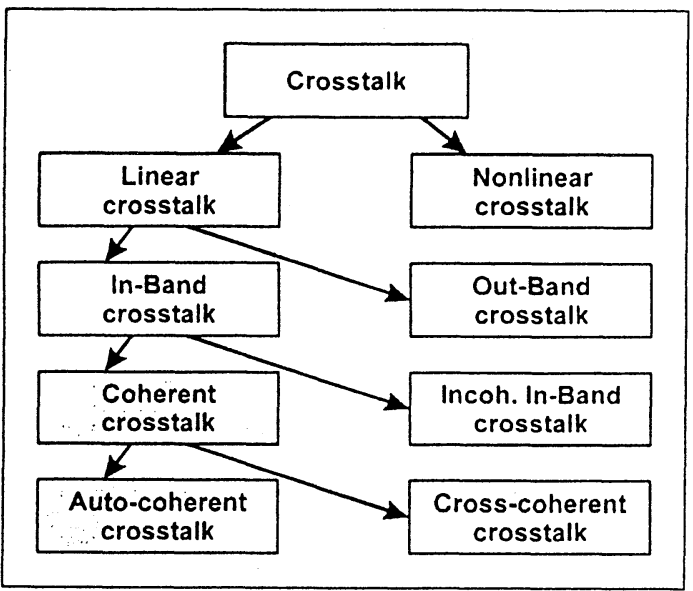

Fig. 6: Crosstalk in WDM Networks

The strongest crosstalk contribution is the "coherent crosstalk". Different optical signals with identical nominal frequencies interfere within the receiver bandwidth (this is the "cross-coherent crosstalk"). A signal which interferes with itself by multipath propagation causes "auto-coherent crosstalk".

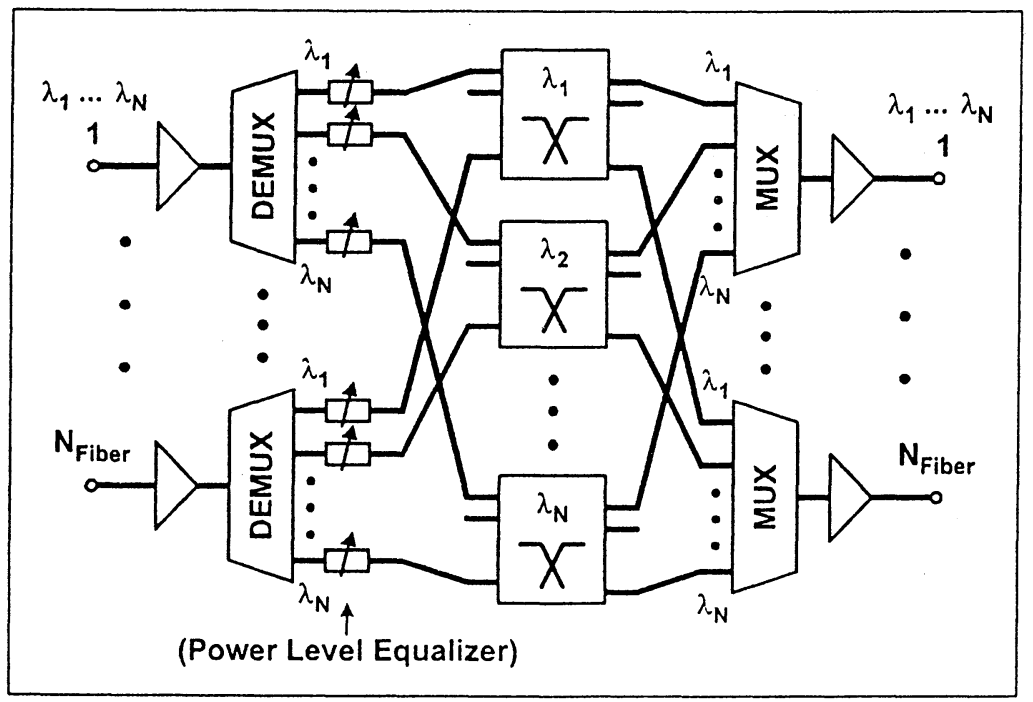

Fig. 7: Wavelength Path Crossconnect

In a simple switching node all wavelength channels from the incoming fibres are first demultiplexed and then switched to the output fibres. This type of node may suffer from a higher degree of blocking probability.

With such nodes the "Wavelength Path" concept can be realised. A better performance may be obtained using the "Virtual Wavelength Path" concept with optical frequency converters within the nodes. Such a node is much more complicated and it is not yet confirmed that the Virtual Wavelength Path concept is superior to the simpler Wavelength Path concept. 
The procedure of evaluating the coherent crosstalk is to figure out all possible spurious paths within the node and then to compare the summation of the spurious signals with the power level of the main path. All demultiplexers, switches and multiplexers contribute to the resulting crosstalk. Finally, the $1 \mathrm{~dB}$-penalty level of crosstalk is obtained by the analysis published by Takahashi.

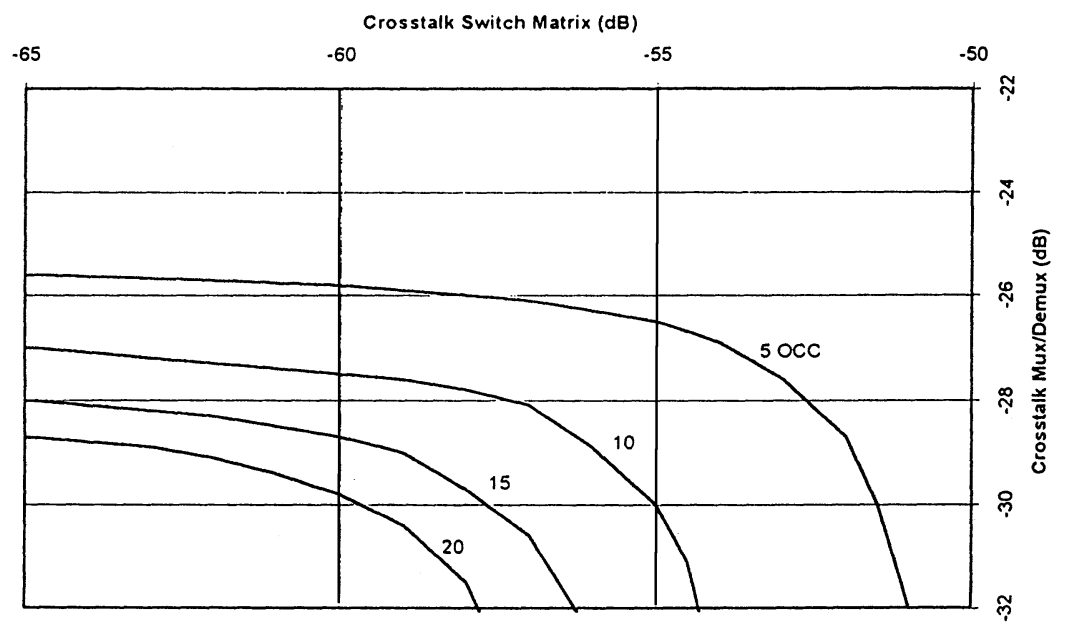

Fig. 8:Tolerable Crosstalk in Crossconnects

Fig. 8 shows the results for a number of cascaded nodes.

The area below the curves shows the region where the power penalty is below $1 \mathrm{~dB}$.

A certain trade-off is possible between the performance of the multiplexers and demultiplexers and the switch-matrices. If space switches with excellent crosstalk performance (of the order of $60 \mathrm{~dB}$ or better) are used, each multiplexer may have down to $27 \mathrm{~dB}$ crosstalk attenuation. If the space stage has a crosstalk attenuation worse than $52 \mathrm{~dB}$, the configuration becomes unsuitable even with excellent multiplexer and demultiplexer performance.

The calculated crosstalk requirements seem realistic. Commercially available multiplexers and demultiplexers exhibit crosstalk levels down to $-30 \mathrm{~dB}$, and crosstalk levels of a matrix based on fibre switches may well be below - $60 \mathrm{~dB}$.

\subsection{Interfaces}

\subsubsection{Client Interfaces}

Interfaces for users are provided at specific ports of network nodes. Users may be either single pieces of equipment, like SDH or PDH equipment, or whole networks. All client interfaces should accept all signals within the limits discussed.

Two different approaches are feasible:

- interfaces are generally usable, i.e. transparent within the constraints shown, or

- interfaces are specialised, e.g. for SDH of specified bitrate.

If unavoidable, "noncompliant" client signals could be made compliant via specific "adapters". Those compliant transmitters would then be part of the network. 
Client interfaces should provide some precautions against erroneous input signals, as regards amplitude and frequency, to avoid disturbances of other signals in the network.

\subsubsection{Transport Interfaces}

Network-side or transport interfaces must be transparently adapted to the parameters of the transmission medium. Their task is

- to provide and accept the appropriate power levels, and

- to care for amplitude and dispersion equalization of the link

\section{Open Issues}

A couple of additional problems have to be solved prior to network implementations.

\subsection{Filter Cascading}

The stability of optical frequencies has a major impact on network design.

An optical sub-path has to traverse several multiplexer and demultiplexer stages. Since the passbands of these filters are not perfectly flat, their cascading leads to an increasing passband narrowing. This is investigated in the simple add/drop ring network shown here.

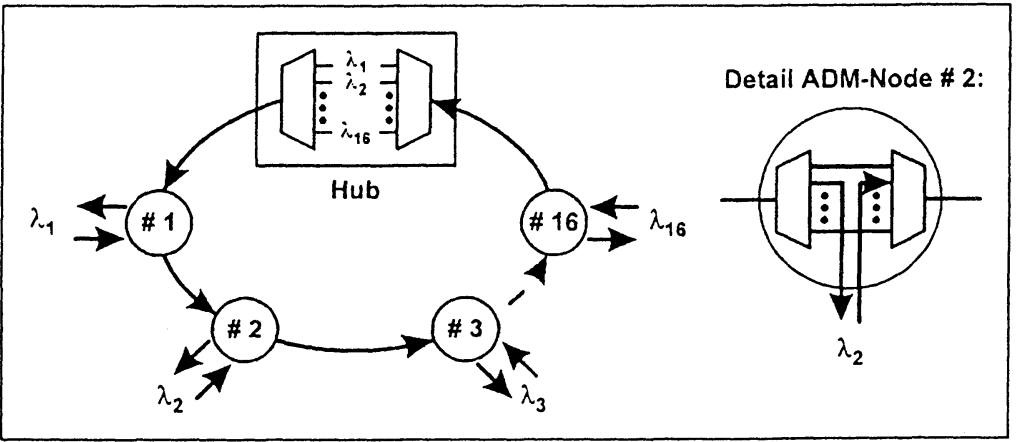

Fig. 9: WDM-Ring with 16 ADM-Nodes

Arrayed waveguide gratings as multiplexers and demultiplexers have a nearly Gaussian passband characteristic. The resulting bandwidth is reduced with the square-root of the number of devices in cascade. In the example shown, the maximum number of cascaded devices equals 32 .

\begin{tabular}{|l|c|}
\hline wavelength channel spacing & $200 \mathrm{GHz}$ \\
\hline $\begin{array}{l}1 \mathrm{~dB} \text { bandwidth of each } \\
\text { multiplexer/demultiplexer }\end{array}$ & $50 \mathrm{GHz}$ \\
\hline $1 \mathrm{~dB}$ bandwidth of a 32 -device cascade & $8.8 \mathrm{GHz}$ \\
\hline suitable for maximum bit rate class & class $2 ; 2.5 \mathrm{Gbit} / \mathrm{s}$ \\
\hline
\end{tabular}

Table 3: WDM-Ring Performance 
In addition to the bandwidth narrowing effect, a second influence can lead to path degradation. If all centre frequencies are not perfectly aligned and if additional short-time fluctuations of the centre frequencies (due to temperature changes, change of polarization states etc.) cannot be excluded, the received optical power fluctuates as shown.

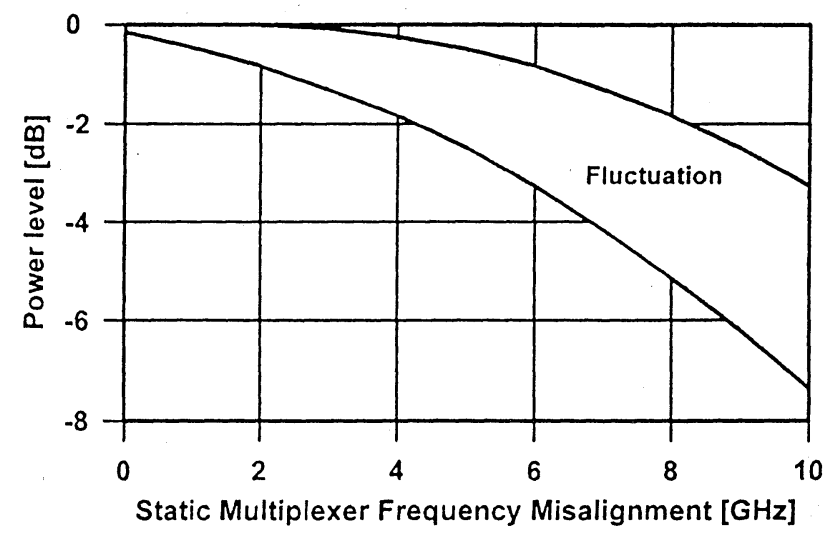

(calculated for dynamic frequency deviation of AWG-filters: $\pm 2 \mathrm{GHz}$ )

Fig. 10: Power Level Fluctuations in a 16-node ADM-Ring

A short-term fluctuation of $2 \mathrm{GHz}$ for each device is assumed. In order to keep the resulting power fluctuations below $1 \mathrm{~dB}$, the static centre frequencies should be aligned to better than $2 \mathrm{GHz}$. The requirements on the frequency accuracy of each laser transmitter are of the same order.

Cascading of filters has a strong impact on path performance.

Therefore

- a flat passband characteristic for multiplexers and demultiplexers is mandatory

- a flat passband may be obtained at the expense of additional insertion loss; this should be acceptable within certain limits.

The impact of filter characteristics and slopes on dispersion has not yet been considered.

\subsubsection{Frequency Referencing}

For reasons already considered it may be necessary to consider frequency referencing techniques.

Possible implementations are

- stabilization by local references or

- locking to distributed absolute reference frequencies within the network.

\subsection{Power level control}

Measures have to be developed to keep the power level at each interface point within specified ranges. The static as well as the dynamic behaviour of power levels ("optical transients") of switched optical paths is an important issue and must be considered. 


\subsection{Monitoring of Optical Path Performance}

Monitoring must be an integral part of the network. Quality performance of active and standby channels must be known and guaranteed for the relevant class. Furthermore, degradation effects should be distinguished and localised.

\subsection{Network Management in Transparent Systems}

Transport systems, such as SDH, have well defined internal means for dealing with faults and performance monitoring, and whatever is done in the optical network must work together with the transport systems. If there is a fault, both the SDH layer and the optical layer will know about it and means must be implemented to co-ordinate their response.

As different transport systems (like SDH and FDDI) may share the same transparent Photonic Network, the network management will have to interwork with different systems.

\subsection{Transport of Management Information}

Transport of monitoring and management information should be provided as an integral part within the network. All types of "overhead" can for example be carried by dedicated WDM channels of each multiplex section.

\section{Conclusion}

These planning guidelines are regarded as first proposals to design a transparent Photonic Network for practical implementation. A "bottom-up" approach based on a reference configuration has been used for determining the maximum number of cascadable sections. Calculations indicate that largescale core networks with high capacity can be realised. Transparent optical sub-paths for signals up to $10 \mathrm{Gbit} / \mathrm{s}$, assembled in a 16-channel multiplex, can bridge distances of $400 \mathrm{~km}$, and even longer distances with lower bitrates. Up to 10 transparent crossconnects are cascadable with ultra-high throughput of 2.56 Terabit/s (16 fibres times 16 wavelengths each transmitting $10 \mathrm{Gbit} / \mathrm{s}$ ). Prerequisites are WDM multiplexers and demultiplexers with sufficiently flat passband characteristics and sufficiently stable transmitters.

Considering the numerical results obtained, it can be concluded that a country of the size of Germany could be covered by a transparent Photonic Network.

\section{References}

[1] Draft ITU-T Recommendation G.otn

Architecture of optical transport networks, Version 1.0 Ipswich, 1996

[2] Draft ITU-T Recommendation G.691

Optical Interfaces for Single Channel SDH Systems with Optical Amplifiers, and STM-64 systems Geneva, May/June 1996

[3] Draft ITU-T Recommendation G.mcs

Optical Interfaces for Multichannel Systems with Optical Amplifiers, June 1996

[4] ITU-T Recommendation G.652

Characteristics of a Single-Mode Optical Fibre Cable

[5] Takahashi, H. et al.

Transmission Characteristic of Arrayed Waveguide NxN Wavelength Multiplexer

Journal of Ligthwave Technology, Vol. 13, No. 3, March 1995, pp. 447-455 